\title{
Experimental and theoretical study of a piezoelectric vibration energy harvester under high temperature
}

\author{
Emmanuelle Arroyo, Member, IEEE, Yu Jia, Member, IEEE, Sijun Du, Student member, IEEE, Shao Tuan Chen, \\ and Ashwin Seshia, Senior member, IEEE
}

\begin{abstract}
This work focuses on studying the effect of increasing the ambient temperature up to $160^{\circ} \mathrm{C}$ on the power harvested by a MEMS piezoelectric micro-cantilever manufactured using an aluminum nitride-on-silicon fabrication process. An experimental study shows that the peak output power decreases by $60 \%$ to $70 \%$ depending on the input acceleration. A theoretical study establishes the relationship of all important parameters with temperature and includes them into a temperature-dependant model. This model shows that around $50 \%$ of the power drop can be explained by a decreasing quality factor, and that thermal stresses account for around $30 \%$ of this decrease.
\end{abstract}

Index Terms-Energy harvesting, high temperature, MEMS, cantilever, AIN.

\section{INTRODUCTION}

$\mathbf{I}$ NERTIAL vibration energy harvesting has seen much interest in recent years, due to the potential of powering lowpower distributed sensor nodes using ambient kinetic energy.

The applications of such distributed sensor nodes, which include industrial machine surveillance, smart infrastructure or structural health monitoring for aerospace and automotive applications, often involve operation under harsh environment conditions such as in high vibrations environments or operation at high temperatures where access to alternative, more conventional energy sources may not be possible. However, there have not been significant previous studies on the high temperature behaviour of inertial vibration energy harvesters.

Within the field of inertial vibration energy harvesters, piezoelectric MEMS based energy harvesters have received significant recent research interest due to the small size and potential for closer integration with the rest of the sensor system [1].

While most piezoelectric materials lose piezoelectric properties when heated up, Aluminium Nitride has been shown to be stable at high temperatures. For instance, the Curie temperature of commonly used piezoelectric material Lead Zirconate Titanate (PZT) is of about $350{ }^{\circ} \mathrm{C}$, leading to a maximum recommended operation temperature of $150{ }^{\circ} \mathrm{C}$ to $250{ }^{\circ} \mathrm{C}$, whereas it reaches more than $2000^{\circ} \mathrm{C}$ for AlN. Transducers using thin films AlN have also been successfully utilised at high temperatures up to $1000{ }^{\circ} \mathrm{C}$ [2], [3].

However, when it is integrated as a thin-film piezoelectric layer on a resonating silicon micro-cantilever, other mechanical or electrical effects induced by temperature can affect the harvested power. In the objective of understanding these effects and their relative proportions, this work, building upon initial results presented in a recent conference paper [4], focuses on experimentally studying the effects of temperatures

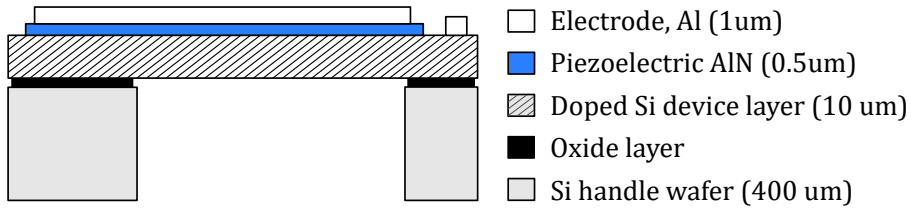

Fig. 1: Schematics of the various layers of the cantilever, due to MEMS fabrication process.

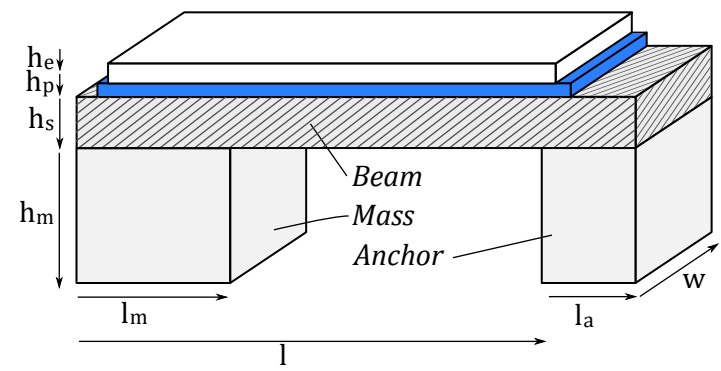

Fig. 2: Schematics of the cantilever, with important dimension parameters.

up to $160{ }^{\circ} \mathrm{C}$ on the power harvested by a classical MEMS energy harvester. In the second part, a model is proposed to explain the harvester behaviour, and the causes behind the power drop in order to predict and anticipate this effect.

\section{EXPERIMENTAL CHARACTERISATION}

\section{A. Tested structure}

The micro-cantilever topology is considered here as this represents the most popular design approach for inertial energy harvesters. The beam is clamped at one end and a proof mass is suspended at the other end to adjust the resonance frequency of the device. The harvester is fabricated using a $0.5 \mu \mathrm{m}$ thick Aluminium Nitride (AlN) piezoelectric layer on $10 \mu \mathrm{m}$ doped Silicon (Si) device layer on a Silicon-On-Insulator (SOI) substrate. Part of the unetched $400 \mu \mathrm{m}$ thick silicon substrate is used as a proof mass. Finally a $1 \mu \mathrm{m}$ Aluminium layer is used as a top electrode (Fig. 1). Fig. 2 shows a drawing of the cantilever with important dimensions summed up in Table I. Four such cantilevers fit into a single $1 \mathrm{~cm} \mathrm{x} 1 \mathrm{~cm}$ die, as shown in Fig. 3, showing a picture of a die mounted in a chip carrier.

\section{B. Experimental setup}

The die is glued to a metal spacer shaped in the form of a ring. The spacer is itself placed inside a socket (shown in 
TABLE I: Dimensions of the tested cantilever

\begin{tabular}{|c||c|}
\hline Parameters & Value $(\mu \mathrm{m})$ \\
\hline$h_{e}$ & 1 \\
$h_{p}$ & 0.5 \\
$h_{s}$ & 10 \\
$h_{m}$ & 400 \\
$l$ & 3600 \\
$l_{m}$ & 2200 \\
$w$ & 3600 \\
\hline
\end{tabular}

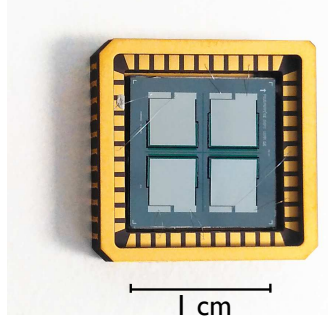

Fig. 3: Picture of the die.

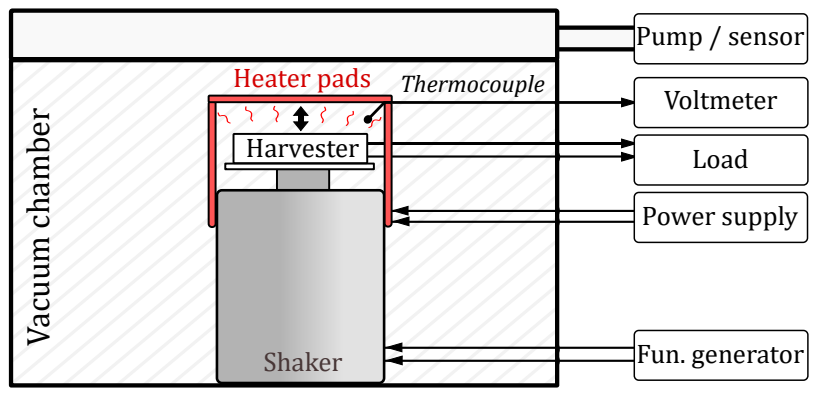

Fig. 4: Schematic diagram of the experimental setup.

Fig. 3) and provides the necessary clearance during vibration. The chip is fitted inside a package which is soldered to a PCB and fixed onto an electromagnetic shaker (LDS V406 M4-CE) driven by a function generator (Agilent Technologies 33250A $80 \mathrm{MHz}$ waveform generator) and an amplifier (LDS PA100E Power Amplifier). The experimental setup is described in Fig. 4. The shaker is placed inside a vacuum chamber regulated at $0.33 \mathrm{mbar}$. The hermetically enclosed chamber ensures operation at constant volume throughout the experiment. Heating pads are used to heat up the device and a thermocouple provides feedback on the temperature close to the harvester. Experiments are performed by sweeping frequency three times at each temperature value and input acceleration: with the device in open circuit, in short circuit and on a matched $300 \mathrm{k} \Omega$ resistive load. At each step the RMS voltages are recorded. Given that the shaker used to fit inside the vacuum chamber is too small to accommodate both the harvester and an accelerometer, there is no real-time feedback on the acceleration. The acceleration level is determined by a separate test on a larger shaker using an accelerometer. The voltage levels of the harvester in open-circuit are compared between the two tests, leading back to the acceleration value. Over the frequency sweeps experiments, the acceleration can be shown to vary by around $1.5 \%$. Therefore it is considered constant and to have negligible effect on the power output.

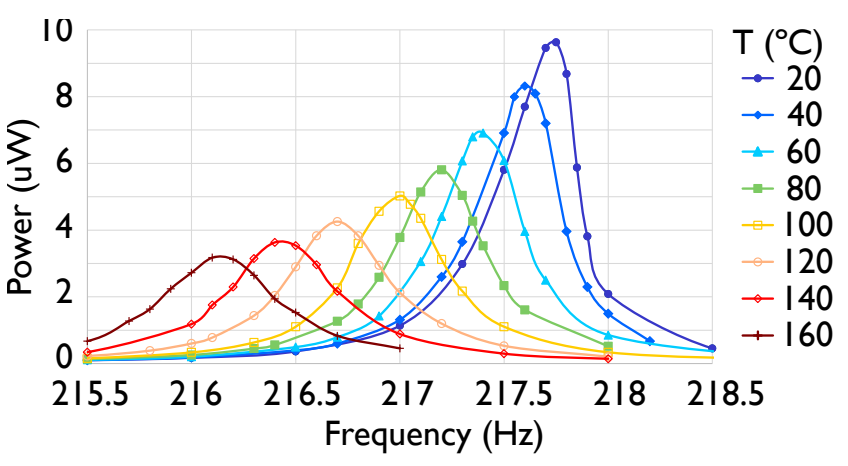

Fig. 5: Experimental result. Power on optimal load as a function of frequency for several temperatures and applied acceleration of $0.4 \mathrm{~g}$.

\section{Experimental results}

1) Harvested power: The power is measured as the power dissipated in the optimal load as a function of the input vibration frequency. The resulting frequency sweeps are plotted in Fig. 5, for several temperatures ranging from $20{ }^{\circ} \mathrm{C}$ to $160{ }^{\circ} \mathrm{C}$. The behaviour shows a slightly nonlinear response when the harvested power is large. It corresponds to cases where the tip displacement amplitude is the largest: at room temperature and driven by higher accelerations. When the temperature increases, the harvested power decreases as well as the non linearity.

2) Characteristic parameters: The maximum power, at resonance, is recorded as a function of the applied temperature for several input accelerations, and plotted in Fig. 6. As shown, there is a decrease in the peak power of around $60 \%$ at $1 \mathrm{~g}$ from 20 to $160{ }^{\circ} \mathrm{C}$, and up to around $70 \%$ at the lower acceleration of $0.4 \mathrm{~g}$.

The resonance frequency, shown in Fig. 7 is independent of the input acceleration and decreases by around $0.7 \%$ over the tested temperature range.

Finally, the mechanical quality factor is calculated from the half power frequency bandwidth, and the results are plotted in Fig. 7 as a function of the applied temperature, for several accelerations. A decrease of around 35\% of the mechanical quality factor is measured, independently of the input acceleration.

As seen, the harvested power decreases by more than $50 \%$ when the temperature is increased to $160{ }^{\circ} \mathrm{C}$, which can significantly impact on the performances of the entire system, especially when considering micro-scale harvesters generating 10 's of micro watts. Therefore, the following section focuses on systematically studying the evolution of the device parameters with temperature, and constructing a suitable predictive model to explain the observations involving a power drop with temperature.

\section{Modelling MEMS HARVESTERS}

Given that the cantilever demonstrates linear response, it is modelled around its resonance frequency as an equivalent linear mass-spring-damper system subjected to an input sinusoidal vibration $y$ (Fig. 8). The induced displacement of the 


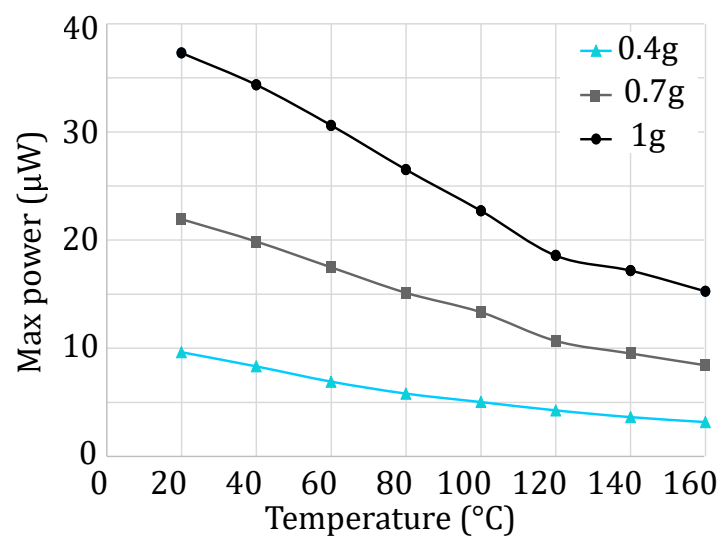

Fig. 6: Experimental results : maximum power as a function of temperature
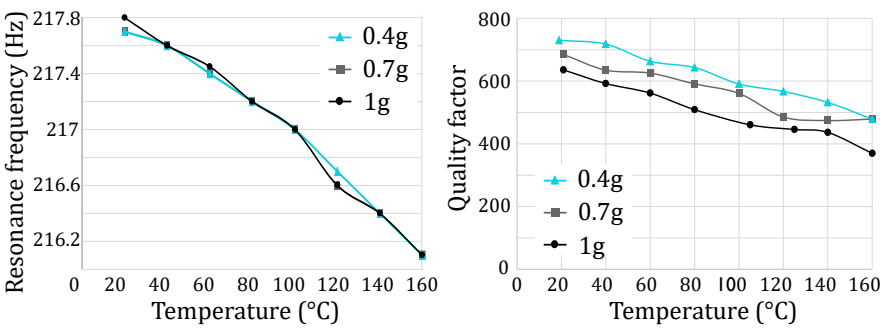

Fig. 7: Experimental results: characteristic parameter variation as a function of temperature

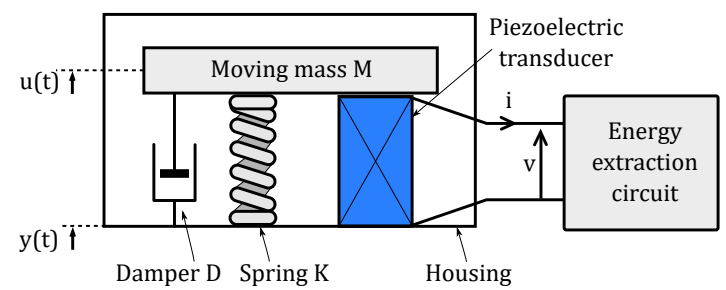

Fig. 8: Mechanical equivalent model of the piezoelectric cantilever.

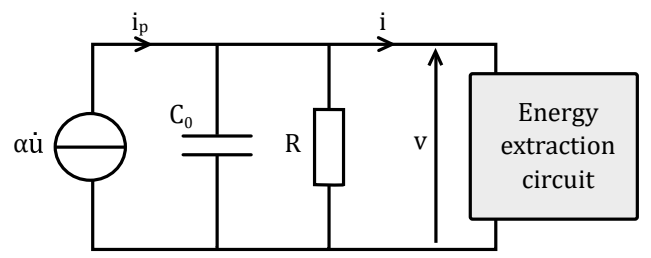

Fig. 9: Electrical equivalent model of the piezoelectric cantilever.

equivalent mass $M$ is $u$, the equivalent stiffness is $K$ and mechanical losses are associated with the damping coefficient $D$. The electrical equivalent circuit of the piezoelectric generator is shown in Fig. 9, where the generated current $i_{p}$ is proportional to the input velocity, $C_{0}$ is the capacitance and $R$ the parasitic resistance of the piezoelectric element. Usually very large, this parasitic resistance can be neglected. Finally, $\alpha$ is the electromechanical coupling coefficient.

The harvester behaviour is described by the two coupled mechanical and electrical equations (1) and (2).
TABLE II: Characteristic parameters

\begin{tabular}{ccl}
\hline Parameters & Expression & Description \\
\hline$k_{m}^{2}$ & $\alpha^{2} /\left(K_{o c} C_{0}\right)$ & Electromechanical coupling coefficient \\
$Q_{m}$ & $\sqrt{K_{s c} M / D}$ & Mechanical quality factor \\
$\xi_{l}$ & $1 /\left(R_{l} C_{0} \omega_{0}\right)$ & Load coefficient \\
\hline
\end{tabular}

TABLE III: Normalisations

\begin{tabular}{ccl}
\hline Parameters & Expression & Description \\
\hline$\underline{t}$ & $\omega_{0} t$ & Time normalisation \\
$y^{\prime}$ & $d y / d \underline{t}=\dot{y} / \omega_{0}$ & Normalisation of the speed \\
$y^{\prime \prime}$ & $d y^{\prime} / d \underline{t}=\ddot{y} / \omega_{0}^{2}$ & Normalisation of the acceleration \\
$\underline{\omega}$ & $\omega / \omega_{0}$ & Normalisation of the pulsation \\
$\underline{\underline{i}}$ & $i /\left(\alpha \omega_{0}\right)$ & Normalised current \\
$\underline{v}$ & $v C_{0} / \alpha$ & Normalised voltage \\
\hline
\end{tabular}

$$
\begin{aligned}
-M \ddot{y} & =M \ddot{u}+K+D \dot{u}+u+\alpha v \\
i & =\alpha \dot{u}-C_{0} \dot{v}
\end{aligned}
$$

When the system is in short circuit, the resonance frequency is defined as:

$$
\omega_{s c}=\sqrt{\frac{K}{M}}
$$

When the system is in open circuit, equation (2) gives the voltage proportional to the displacement, leading to a modified stiffness and hence a resonance frequency given by equation (4).

$$
\omega_{o c}=\sqrt{\frac{K+\frac{\alpha^{2}}{C_{0}}}{M}}
$$

The characteristic parameters listed in Table II and the normalisations shown in Table III are introduced. A global coupling coefficient, representing the efficiency between the electrical and mechanical domains, is often used. It is defined as shown in Table II, and can also be calculated from the open circuit and short circuit resonance frequencies (5). A modified coupling coefficient $k^{2}$ is defined as $k^{2}=k_{m}^{2} /\left(1-k_{m}^{2}\right)$ to simplify expressions.

$$
k_{m}^{2}=\frac{1 / 2 C_{0} v^{2}}{1 / 2 K_{o c} u^{2}}=\frac{\omega_{o c}^{2}-\omega_{s c}^{2}}{\omega_{o c}^{2}}=\frac{\alpha^{2}}{K_{o c} C_{0}}
$$

Replacing these parameters in Equations (1) and (2) yields the final normalised expressions given in (6) and (7). Details of this normalisation can be found in [5].

$$
\begin{aligned}
-y^{\prime \prime} & =u^{\prime \prime}+\frac{u^{\prime}}{Q_{m}}+u+k_{m}^{2} \underline{v} \\
\underline{i} & =u^{\prime}-v^{\prime}-2 \xi_{e} \underline{v}
\end{aligned}
$$

When the system is connected to a resistive load $R_{l}$, the current expression, calculated as $i=v / R_{l}$, is replaced into (7). The variables are translated into complex variables to solve the normalised voltage amplitude $\underline{v}_{M}$ expression as a function of the displacement amplitude $u_{M}$ (8). Using (6), $u_{M}$ can then be expressed as a function of the various parameters and the power dissipated into the load resistance is calculated from the squared displacement amplitude using (9). 


$$
\begin{gathered}
\underline{v}_{M}^{2}=\frac{\underline{\omega}^{2}}{\underline{\omega}^{2}+4 \xi_{l}^{2}} u_{M}^{2} \\
\underline{P}=\frac{8 \xi_{l} k^{2}}{\gamma_{M}^{2} Q_{m}} \frac{\underline{\omega}^{2}}{\underline{\omega}^{2}+4 \xi_{l}^{2}} u_{M}^{2}
\end{gathered}
$$

The normalised power expression calculated from (9) only depends on the characteristic parameters defined in Table II. When the coupling coefficient is increased, the power increased accordingly until reaching a limit power $P_{l i m}$, which depends on the equivalent mass of the system, on the input acceleration amplitude $\gamma_{M}$, or the resonance angular frequency $\omega_{0}(10)$.

$$
P_{l i m}=\frac{M \gamma_{M}^{2} Q_{m}}{8 \omega_{0}}
$$

In order to express the influence of temperature on the harvested power, the dependency of each parameter on temperature is investigated in the following section.

\section{TEMPERATURE-DEPENDENT PARAMETERS}

\section{A. Material parameters}

Three different materials are stacked to form the cantilever. This includes the silicon device layer, the aluminium nitride piezoelectric layer and the aluminium electrode layer. The silicon layer having the largest thickness by far, it is the dominant determinant of the overall mechanical properties of the stack. Important parameter values at ambient temperature are recapitulated in Table IV.

Literature studies show that the material parameters evolutions with temperature can be described by a linear relationship in a first approach. Therefore, when the temperature is increased to a value $\mathrm{T}$, the new parameter $\operatorname{Param}(T)$ is calculated from the parameter value at ambient temperature $\operatorname{Param}_{0}$ and from the temperature coefficient defined as: $\frac{1}{\text { Param }_{0}} \frac{d \text { Param }}{d T}$. The temperature coefficient of each parameter and material is extracted from literature studies. When the behaviour of the cantilever as a whole is considered, equivalent parameters referring to the stack of materials are used. They are named Param $_{e q}$ and their expression is given in Table IV as well.

1) Young modulus: The decrease of the silicon Young modulus as a function of temperature has been experimentally measured by several studies reporting temperature coefficients at around: $\frac{1}{E} \frac{d E}{d T}=-80 \cdot 10^{-6}{ }^{\circ} \mathrm{C}^{-1}$ [6] [7]. Literature studies give a temperature coefficient of around $-51 \cdot 10^{-5 \circ} \mathrm{C}^{-1}$ for aluminium [8] and the value of $-48 \cdot 10^{-6 \circ} \mathrm{C}^{-1}$ is taken for AlN [9].

2) Poisson coefficient: Very few studies report on the evolution of the silicon Poisson coefficient versus temperature. A NASA report from 1973 [10] gives the variation of Poisson's ratio for single-crystal silicon as $\nu=0.197-0.000095 T$ with $\mathrm{T}$ the temperature in ${ }^{\circ} \mathrm{C}$. This leads to the following

\begin{tabular}{|c|c|c|}
\hline Silicon & & \\
\hline$\rho_{s 0}$ & $2330 \mathrm{~kg} / \mathrm{m}$ & Si density \\
\hline$E_{s 0}$ & $169 \cdot 10^{9} \mathrm{~Pa}$ & Si Young modulus \\
\hline$\alpha_{[110]}$ & $2.6 \cdot 10^{-6}$ & Si thermal exp. coeff. $x, y$ plane \\
\hline$\alpha_{[100]}$ & $0.7 \cdot 10^{-6}$ & Si thermal exp. coeff. along $\mathrm{z}$ \\
\hline$\kappa$ & $149 \mathrm{~W} / \mathrm{m} / \mathrm{K}$ & Thermal conductivity \\
\hline$C_{p}$ & $700 \mathrm{~J} / \mathrm{kg} / \mathrm{K}$ & Specific heat \\
\hline \multicolumn{3}{|c|}{ Aluminium Nitride } \\
\hline$\rho_{p 0}$ & $3260 \mathrm{~kg} / \mathrm{m}$ & AlN density \\
\hline$E_{p 0}$ & $320 \cdot 10^{9} \mathrm{~Pa}$ & AlN Young modulus \\
\hline$\epsilon_{r 0}$ & 9 & AlN relative permeability \\
\hline$\alpha_{p 0}$ & $4.5 \cdot 10^{-6}$ & AlN linear thermal exp. coeff. \\
\hline \multicolumn{3}{|c|}{ Aluminium } \\
\hline$\rho_{a 0}$ & $2698 \mathrm{~kg} / \mathrm{m}$ & Al density \\
\hline$E_{a 0}$ & $69 \cdot 10^{9}$ & Al Young modulus \\
\hline$\alpha_{a 0}$ & $23.1 \cdot 10^{9}$ & Al linear thermal exp. coeff. \\
\hline \multicolumn{3}{|l|}{ Stack } \\
\hline$h_{e q}$ & $h_{p 0}+h_{a 0}+h_{s 0}$ & Stack height \\
\hline$\rho_{e q}$ & $\frac{\rho_{p 0} h_{p 0}+\rho_{a 0} h_{a 0}+\rho_{s 0} h_{s 0}}{h_{e q}}$ & Stack Density \\
\hline$E_{e q}$ & $\frac{E_{p 0} h_{p 0}+E_{a 0} h_{a 0}+E_{s 0} h_{s 0}}{h_{e q}}$ & Stack Young modulus \\
\hline
\end{tabular}
temperature coefficient: $\frac{1}{\nu} \frac{d \nu}{d T}=-48 \cdot 10^{-5 \circ} \mathrm{C}^{-1}$.
TABLE IV: Materials parameters at ambient temperature

3) Density: Silicon density is reported to have the following temperature dependency (11), with $T_{k}$ the temperature in Kelvin [11]. It gives a temperature coefficient of $-9.4 \cdot 10^{-6 \circ} \mathrm{C}^{-1}$.

$$
\rho_{s}(T)=2.33-2.19 \cdot 10^{-5} T_{k}
$$

4) Relative permittivity: The relative permittivity of AlN is reported to increase with temperature with a temperature coefficient of $1.4 \cdot 10^{-4 \circ} \mathrm{C}^{-1}[12]$.

5) AlN Piezoelectric coefficient: A study by Kano et al. published in 2006 showed that the measured value of the piezoelectric d33 coefficient of AlN deposited on Si had the constant value of $1.38 \mathrm{pC} / \mathrm{N}$ at temperatures ranging from $20^{\circ} \mathrm{C}$ to $300{ }^{\circ} \mathrm{C}$ [13].

6) Thermal expansion: The thermal expansion of materials subjected to an increase of temperature induces variation in the cantilever dimensions, therefore affecting the dynamics. The variation in dimensions are calculated using the thermal expansion coefficients for silicon: $\alpha_{[110]}$ and $\alpha_{[100]}$, corresponding to the length and thickness of the layer respectively, taken from [14]. The new dimension at a temperature $\mathrm{T}$ in ${ }^{\circ} \mathrm{C}\left(\operatorname{Dim}_{T}\right)$ is calculated from the initial dimension $\operatorname{Dim}_{0}$ as shown in equation (12).

$$
\operatorname{Dim}(T)=\operatorname{Dim}_{0}(1+\alpha(T)(T-25))
$$

7) Air viscosity: the dynamic viscosity of air $\mu$ has an influence on the damping of the cantilever when the latter is operating in air. Sutherland's formula is used to derive the dynamic viscosity of air as a function of the temperature (13).

$$
\mu(T)=\frac{1.512 \cdot 10^{-6} T^{3 / 2}}{T+120}
$$


8) Heat capacity at constant pressure $C_{p}$ : The silicon molar heat capacity at constant pressure is linked to the temperature in Kelvin $T_{k}$ by equation (14). Expressing temperature in Celsius units and dividing by the silicon molar mass leads to the expression of $C_{p}$ as a function of temperature given in (16).

$$
\begin{array}{r}
C_{p m}\left(T_{k}\right)=23.5+3.05 \cdot 10^{-3} T_{k}-2.93 \cdot 10^{-7} T_{k}^{2} \\
C_{p m}(T)=24.311+2.89 \cdot 10^{-3} T-2.93 \cdot 10^{-7} T^{2} \\
C_{p}(T)=865.607+102.9 \cdot 10^{-3} T-10.43 \cdot 10^{-6} T^{2}
\end{array}
$$

\section{B. Resonance frequency}

The relationships between material parameters and temperature established in the previous section are now used to study the evolution of characteristic parameters linked with the dynamic behaviour of the cantilever and therefore linked to the harvested power.

The basic relationship to express the cantilever resonance frequency is given in (17) as the square root of the beam stiffness $K$ divided by the equivalent mass $M$. Those two parameters can in turn be defined as a function of the material parameters and cantilever dimensions.

$$
\omega_{0}=\sqrt{\frac{K}{M}}=\sqrt{\frac{3 E I}{M l^{3}}}
$$

Where $l$ is the active length of the cantilever, $E$ is the equivalent Young modulus of the structure and $I$ the area moment of inertia, approximated as the moment of inertia of a single layer silicon cantilever (18). This assumption is driven by the fact that the silicon device layer is approximately 10 times thicker than the other ones. The equivalent mass of a plain cantilever beam with a proof mass $m_{l}$ and significant beam mass $m_{b}$ is approximated by (20) [15].

$$
\begin{aligned}
I & =\frac{w\left(h_{s}+h_{p}+h_{e}\right)^{3}}{12} \\
M & =0.2427 m_{b}+m_{l} \\
& =\rho_{s} w\left(0.2427 h_{s} l_{e q}+h_{m} l_{m}\right)
\end{aligned}
$$

The resonance frequency calculated from equation (17) is $228.3 \mathrm{~Hz}$ at ambient temperature, compared to $217.7 \mathrm{~Hz}$ experimentally measured. The difference between the values is mainly due to fabrication tolerance, and over-etching of the mass. When the temperature is increased to $150{ }^{\circ} \mathrm{C}$, theoretical and experimental results are shown in Fig. 10, with the parameters normalised by their maximum values in order to compare them easily. The resonance frequency decreases by $0.69 \%$ which is very similar to experimental results at $0.71 \%$.

Calculation without taking into account the material expansion shows that the frequency evolution is still well described by the Young modulus variation, which alone accounts for a decrease of $0.65 \%$, hence can explain alone the frequency shift.

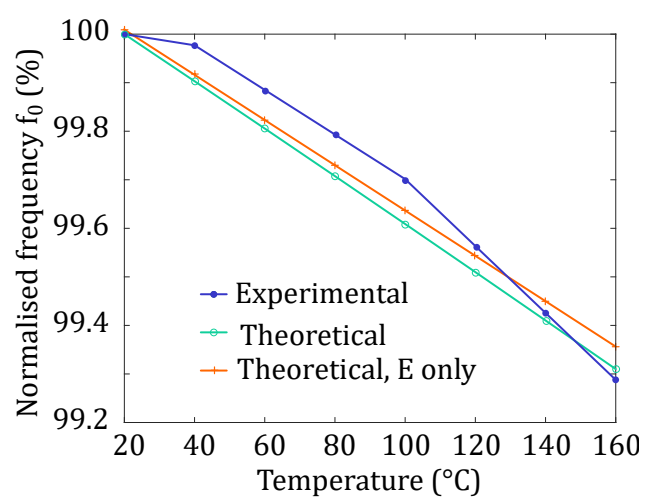

Fig. 10: Calculated and experimental resonance frequency as a function of the applied temperature

\section{Mechanical quality factor}

The mechanical losses in a micro-scale device are a combination of several effects. The most well-known energy loss mechanisms in silicon-based MEMS resonator are the losses induced by air flow including squeeze-film effects, thermoelastic dissipation (TED), and anchor losses. Surface losses are also considered for nanometric scale devices, where the surface to volume ratio becomes sufficiently high. Its influence is considered to be negligible here.

The overall quality factor can be written as the combination of those effects, as in (21).

$$
\frac{1}{Q}=\frac{1}{Q_{\text {air }}}+\frac{1}{Q_{\text {squeeze }}}+\frac{1}{Q_{T E D}}+\frac{1}{Q_{\text {anchor }}}
$$

1) Air damping: In the case of a micro-scale structure operating in low pressure environment, air damping is due to kinetic gas particle collisions. Blom et al [16] provide the expression given in (22) to calculate the damping in that case. From around $200 \mathrm{~Pa}$ and above, the viscosity of the gas determines the air drag, and from the same reference we can approximate the quality factor in that case to the expression given in (23). These two expressions do not take into account any squeeze film effects.

$$
\begin{aligned}
Q_{\text {lowpressure }} & =\frac{1.875^{2} h_{e q}^{2} \sqrt{9 \pi R \rho_{e q} E_{e q}}}{l^{2} \sqrt{384 M_{m o l}}} \frac{\sqrt{T_{k}}}{P} \\
Q_{\text {highpressure }} & =\frac{h_{e q}^{2} \sqrt{E_{e q} \rho_{e q}}}{12 \sqrt{3} \pi l \mu(T)}
\end{aligned}
$$

$\mathrm{R}=8.3144621 \mathrm{~J} / \mathrm{mol} / \mathrm{K}$ is the gas constant, $M_{m o l}$ is the air molecular mass $\left(M_{m o l}=0.028964 \mathrm{~kg} / \mathrm{mol}\right.$ at room temperature), $T_{k}$ is the temperature in $\mathrm{K}, \mu$ the dynamic viscosity of air and $\mathrm{P}$ is the pressure.

The cantilever is first encapsulated in a sealed package or in the vacuum chamber for experiments at a pressure $P_{0}$. Once vacuum sealed, we assume that the package volume $V_{0}$ and number of mole of gas inside it remain constant. Assuming that the gas behaves like a perfect gas, the pressure inside the package is going to vary proportionally with applied temperature, according to the perfect gases law (24). 


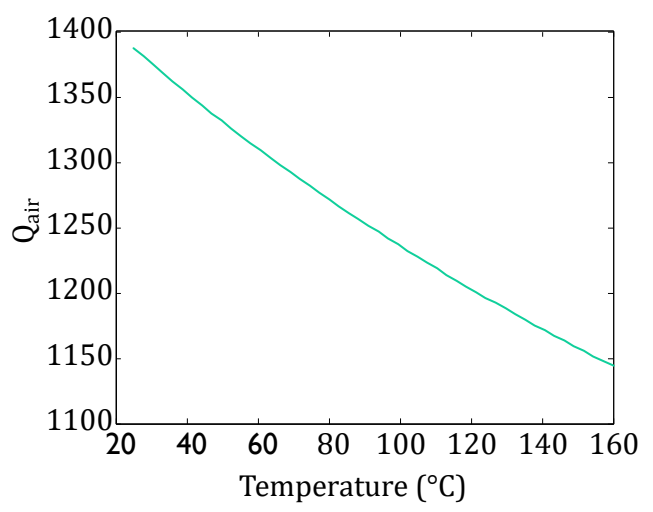

Fig. 11: Calculated quality factor due to air damping, as a function of temperature

$$
P=\frac{n_{0} R}{V_{0}} T_{k}
$$

with $n_{0}$, the number of moles of air encapsulated in the package, calculated from ambient temperature and initial pressure values (25):

$$
n_{0}=\frac{P_{0} V_{0}}{R T_{0}}
$$

Finally, (26) gives the mechanical quality factor expression due to air damping as a function of the temperature inside the package, for a MEMS encapsulated structure. This type of damping is inversely proportional to the root square of the temperature (27). Its theoretical evolution is shown in Fig. 11.

$$
\begin{gathered}
Q_{\text {air }}(T)=\frac{1.875^{2} h_{e q}(T)^{2} \sqrt{9 \pi R \rho_{e q}(T) E_{e q}(T)}}{P_{0} l(T)^{2} \sqrt{384 M_{m o l}}} \frac{T_{0}}{\sqrt{T_{k}}} \\
Q_{\text {air }} \propto \frac{1}{T^{1 / 2}}
\end{gathered}
$$

2) Squeeze film effects: In addition to air damping, squeeze film effects can induce significant damping in MEMS resonating structures vibrating in proximity to a substrate. The pressure in the film is usually calculated using the wellknown Reynolds equation, or simulated using finite elements software. Fig. 12 shows a cross-sectional view of one of the 4 cantilevers pictured in Fig. 3. The mass is moving towards the bottom of the package, leaving a gap $h_{0}$, and $g_{0}$ is the lateral distance to the package. It is supposed that the cantilever length is much larger than its width and thickness in order to consider a 2D model and simplify calculations. It is also assumed that pressure doesn't vary in z-direction (small amplitude deflection).

The estimation of the damping due to squeeze film effects in these conditions is given in (28). Gas rarefaction effects are taken into account by using a modified effective viscosity $\mu_{e f f}$, defined as (29), and Veijola's surface extension model is used to account for border effects (30). If a lid is used to close the package, a similar effect will take place on top of the mass, multiplying the damping by a factor 2 .

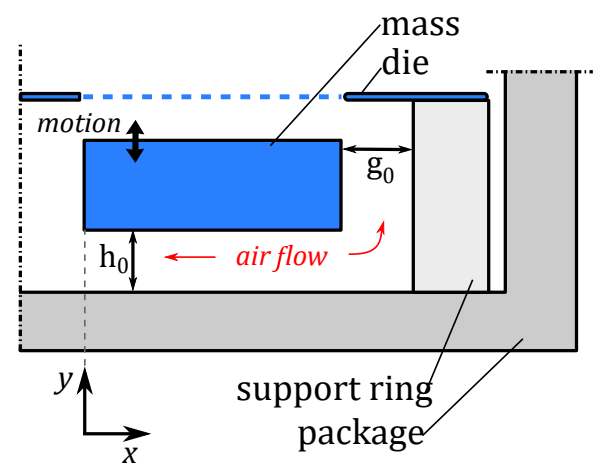

Fig. 12: Schematics of the model used to calculate squeeze film air damping

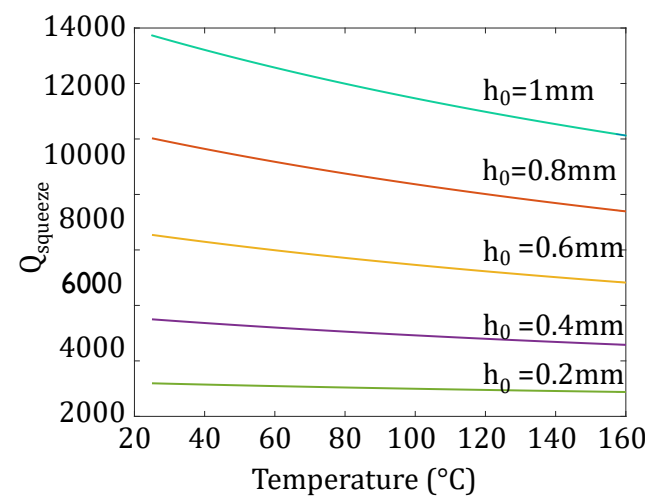

Fig. 13: Calculated quality factor due to squeeze film effects for several air gap dimensions, as a function of temperature.

$$
\begin{gathered}
Q_{\text {squeeze }}=\frac{h_{0}^{3}}{2 \mu_{e f f} l_{m} w^{* 3}} \\
\mu_{\text {eff }}=\frac{\mu(T)}{K_{n}\left(1+9.638 K_{n}^{1.159}\right)} \\
w^{*}=w+0.81\left(1+0.94 K_{n}\right) h_{0}
\end{gathered}
$$

where $K_{n}$ the Knudsen number is defined as the gas mean free path $\lambda$ divided the film thickness $h$.

Even if the width and length of the mass are slightly modified when the temperature increases, the main influence here is given by the effective viscosity, proportional to the air viscosity, itself dependent on the temperature as given in (13). Therefore, the damping due to squeeze film effect will roughly vary with temperature according to (31). Its evolution is shown in Fig. 13 for several values of air gap between the mass and the bottom of the package.

$$
Q_{\text {squeeze }} \propto \frac{120+T}{T^{3 / 2}}
$$

3) Thermoelastic damping: Thermoelastic damping (TED) is linked to thermal losses induced by the strain field of the vibrating structure. Its expression is given by equation (32) [17]. Its evolution with temperature is roughly in $1 / T$ (33).

$$
Q_{T E D}(T)=\frac{\pi^{4} \kappa^{2}+\omega^{2} \rho^{2} C_{p}^{2} w^{4}}{\pi^{2} w^{2} \alpha^{2} T E \kappa \omega}
$$




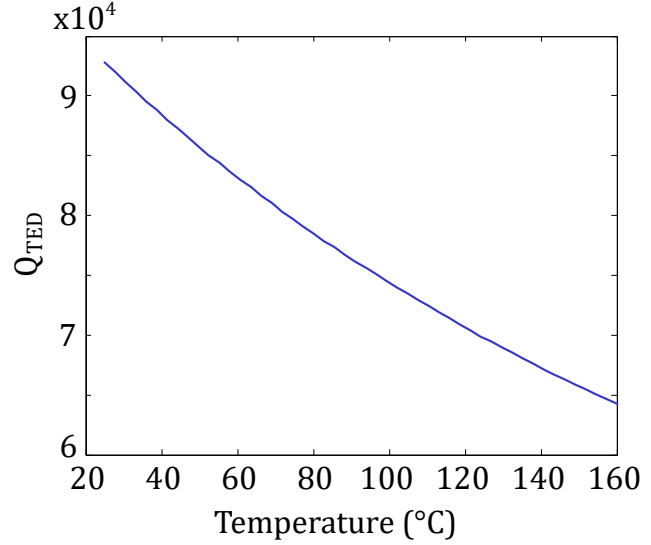

Fig. 14: Calculated quality factor due to thermoelectric damping, as a function of temperature.

$$
Q_{T E D} \propto \frac{1}{T}
$$

Fig. 14 shows the value calculated for the silicon layer as a function of the temperature. The quality factor is inversely proportional to the temperature. As shown, the maximum values are around 90000 and minimum values higher than 60000 , which are around 100 times higher than measured overall quality factors. This suggest that in our case of lowfrequency operating beams, the thermoelastic effects are very small in comparison with other damping effects, and can therefore be neglected.

4) Anchor losses: Finally, the last important effect of damping is anchor losses due to elastic waves propagating from the vibrating cantilever to the support.

Using the model provided in [18] and the dimensions of the beam we consider here, the calculated value for $Q_{\text {support }}$ over the temperature range is in the orders of $10^{7}$, and can therefore safely be neglected here.

5) Global damping: Finally, the global quality factor calculated from each contribution according to (21) is computed and simulated as a function of applied temperature. The results plotted in Fig. 15 show a fairly good estimation of the measured quality factor, as well as a comparable evolution with temperature with a total decrease over the temperature range of around $20 \%$.

This result also confirms that the air damping contributes the most to the damping, even when the device is working under less than $1 \mathrm{mbar}$ pressure. The rate of decrease with temperature is strongly accentuated by the squeeze film effects. It is therefore of importance to operate at lower pressure and further away from the walls, in order to reduce the negative effects of increased temperature on the damping of the device.

\section{Coupling coefficient}

The coupling coefficient $k^{2}$, previously defined as shown in Table (II) can alternatively be expressed as a function of the material parameters (34). The $d_{31}$ coefficient of AlN is taken at $1.36 \mathrm{pC} / \mathrm{N}$ [19], leading to a value of $k^{2}$ at ambient temperature of 0.048 , decreasing to around 0.047 at $150{ }^{\circ} \mathrm{C}$.

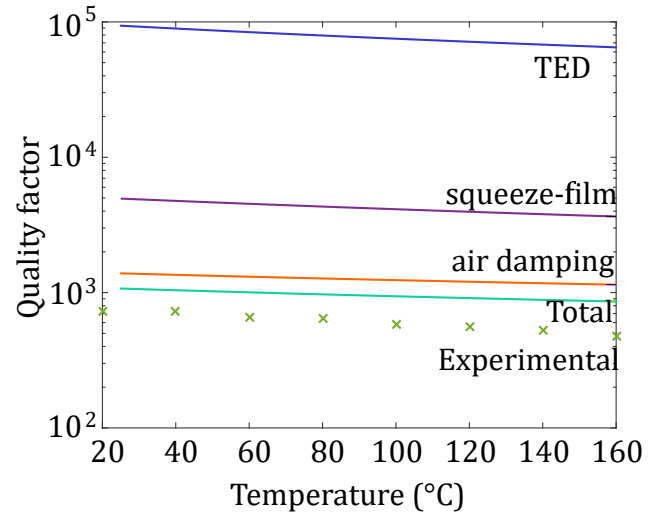

Fig. 15: Total calculated damping contributions from several effects, and experimentally measured damping as a function of temperature. The squeeze film effect is calculated for an air gap of $1 \mathrm{~mm}$.

$$
k^{2}=\frac{d_{31} E_{p}}{\epsilon_{r}}
$$

\section{E. Electrical parameters}

The capacitance of the piezoelectric cantilever can be calculated from the electrode dimensions, the permittivity of free space $\epsilon_{0}$, the temperature-dependant permittivity of AIN $\epsilon_{r}$, the electrodes area over the distance between electrodes, as given in (35). It is an increasing function of the temperature, starting from $1.94 \mathrm{nF}$ at ambient temperature and reaching $1.99 \mathrm{nF}$ at $150{ }^{\circ} \mathrm{C}$, whereas a value of $2.36 \mathrm{nF}$ is experimentally measured at ambient temperature.

$$
C_{0}=\epsilon_{r} \epsilon_{0} \frac{w l}{h_{p}}
$$

Note that current leakage has not been taken into account in this study. Previous studies have shown that there is an exponential increase of the current leakage density with temperature, which might lead to significant decrease in the leakage resistance of the piezoelectric element (neglected in that study) with temperature[20].

\section{F. Thermal stress}

Each material composing the cantilever has a different thermal coefficient. When subjected to increasing temperature, the mismatched dilatations induce stresses on the surfaces. The Finite elements software Comsol is used to simulate these thermal stresses. The cantilever is simulated in 2D, with coupled thermal and mechanical domains. Fig. 16 shows the resulting stress distribution when the cantilever is subjected to $150{ }^{\circ} \mathrm{C}$. The thermal expansion coefficient of the metal layer being the largest, followed by the AlN one and finally the silicon one which is the smallest, an increase in temperature induces a downward curling of the cantilever and large stresses at the border between the layers. The average stress $\sigma$ induced in the piezoelectric layer is evaluated for several input temperatures; its variation is shown in Fig. 17. 


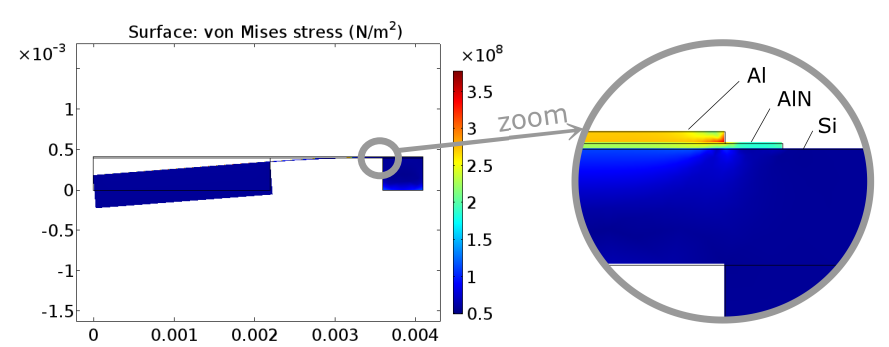

Fig. 16: Comsol simulation of the thermal stresses when the cantilever is subjected to $150^{\circ} \mathrm{C}$, and average stress as a function of the temperature.

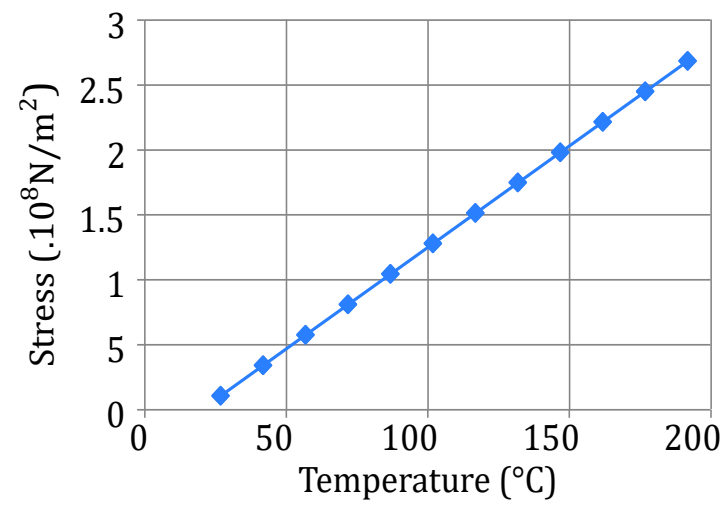

Fig. 17: Comsol simulation of the thermal stresses when the cantilever is subjected to $150{ }^{\circ} \mathrm{C}$, and average stress as a function of the temperature.

\section{Global MOdEL With TEMPERATURE EFFECTS}

Using all the previously described temperature relationships, it is possible to rewrite the classical model equations taking into account the working temperature, leading to the seminormalised equations (36) and (37).

$$
\begin{aligned}
\ddot{y}+\frac{\sigma^{*}(T)}{M(T)} & =\ddot{u}+\frac{\dot{u}}{Q_{m}(T)}+\omega_{0}(T)^{2} u+\frac{\alpha}{M(T)} v \\
i & =\alpha \dot{u}-C_{0}(T) \dot{v}
\end{aligned}
$$

The materials parameters variation with temperature and the temperature dilatation are included in the model, as well as the damping variation experimentally measured.

The thermal stresses are added to the model by means of a global force $\sigma^{*}(T)=\sigma(T) A$, where $A$ is the active piezoelectric area, and $\sigma(T)$, is the stress defined by the Comsol simulation shown in Fig. 17.

\section{A. Resolution}

Those two equations are translated into complex variables and solved by first extracting the displacement amplitude $u_{M}$ to then calculate the power, function of $u_{M}^{2}$. The resolution is the same as the classical model except for the added term $\frac{\sigma^{*}(T)}{M(T)}$ to the displacement expression.

The final calculated power expression is a function of the temperature, the characteristic parameters, the input vibration characteristics like its acceleration and frequency, and the load resistance.

\section{B. Simulation results}

As the parameter variations with temperature have already been studied in the previous section, only the power is studied here. The maximum power over both frequency and load resistance is extracted at each temperature. The acceleration is also changed from $1 \mathrm{~g}$ to $0.4 \mathrm{~g}$ to match with experimental results. The power evolution as a function of applied temperature is plotted in Fig. 18, in dotted lines. As with experimental results (shown in plain lines), the power drop is less important at higher accelerations. The rate of decrease is however slightly different between simulation and experiments: for instance the model predicts a maximum power drop of around $53 \%$ at $0.4 \mathrm{~g}$, while in practical it has been measured at $67 \%$. Given that the calculated parameters show an evolution with temperature very close to the experimental results, the difference can be linked to the assumption that the piezoelectric coefficient is constant over the temperature range. Other effects such as the onset of non-linearity observed at large excitation levels have not been taken into account in this model.

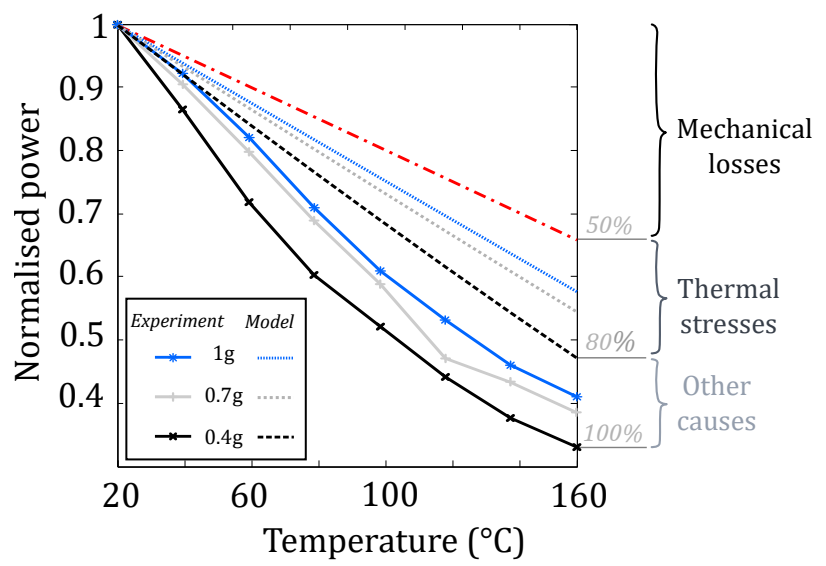

Fig. 18: Simulation of the maximum power as a function of the temperature, when only damping dependency on temperature is taken into account (red dotdashed line), and when all effects are taken into account (dotted lines). Normalised experimental results for each acceleration are also superimposed, in plain lines.

Fig. 19 presents the same results as a function of the applied acceleration. It shows that the model accurately describes the evolution of the power drop with the input acceleration, taking into account the fact that at higher accelerations, the influence of the thermal stresses becomes less significant in comparison with friction effects embodied by the mechanical quality factor.

\section{CONCLUSiON}

This paper studies the effect of increased temperature up to $160{ }^{\circ} \mathrm{C}$ on a MEMS piezoelectric cantilever for energy harvesting. An experimental study showed that the power 


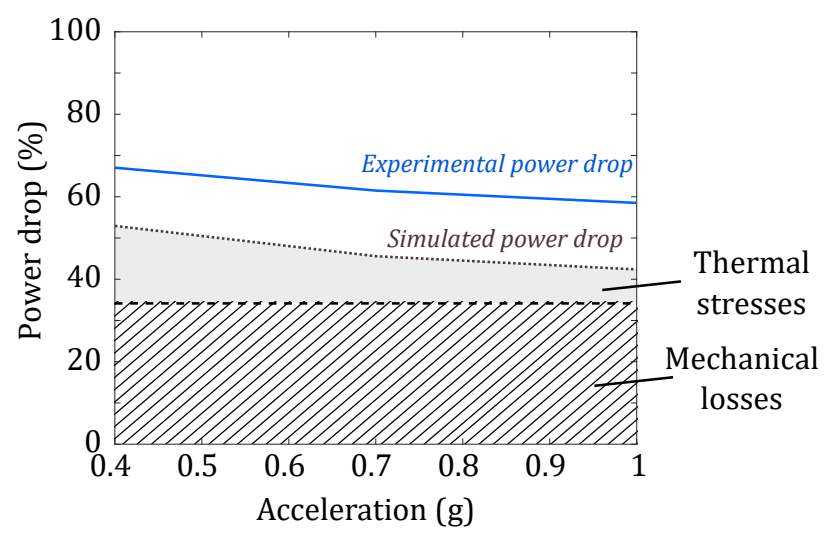

Fig. 19: Power drop versus input acceleration. The plain blue line shows the experimentally measured power drop, the dashed area is the proportion due to mechanical losses according to the model, the plain filled area the proportion due to thermal stresses.

drops by $60 \%$ to $67 \%$ depending on the input acceleration, therefore possibly compromising the performances of the harvester. Each characteristic parameter describing the behaviour of a resonant harvester is described and simulated taking into account the temperature influence on the materials parameters, the thermal expansion and thermal stresses on the cantilever. Their analytical calculations and simulations are in good agreement with the experimental results. A model with temperature is established, allowing the simulation of the maximum power as a function of applied temperature and input acceleration. Results show that a significant part of the power drop (around 50\%) can be explained by the decrease of the mechanical quality factor with increasing temperature. Thermal stresses due to mismatched expansion coefficient in the composite cantilever account for around $30 \%$ of that power drop. Finally, the model accurately describes the evolution of the power output with input acceleration, showing that at lower acceleration levels the thermal stresses become preponderant.

From these results, some solutions can be proposed to try and reduce the effect of temperature on the harvested power by a piezoelectric cantilever. Patterning of the top electrode could help reduce the thermal stresses for instance.

\section{REFERENCES}

[1] Anton S R and Sodano H A 2007 Smart Materials and Structures 16 R1-R21 ISSN 0964-1726

[2] Tittmann B R, Parks D A and Zhang S O 2013 13th International Symposium on Nondestructive Characterization of Materials (NDCMXIII) (Le Mans, France)

[3] Darnjanovic D 1998 Current Opinion in Solid State \& Materials Science 3 469-473

[4] Arroyo E, Jia Y, Du S, Chen S T and Seshia A 2016 PowerMEMS 2016 vol 773 ISSN 1742-6588

[5] Arroyo E, Badel A, Formosa F, Wu Y and Qiu J 2012 Sensors and Actuators A: Physical 183 148-156 ISSN 09244247

[6] Cho C H 2009 Current Applied Physics 9 538-545 ISSN 15671739

[7] Jeong J H, Chung S H, Lee S H and Kwon D 2003 Journal of microelectromechanichal systems $\mathbf{1 2}$

[8] McLellan R B and Ishikawa T 1987 Journal of Physics and Chemistry of Solids 48 603-606 ISSN 00223697

[9] Bruls R, Hintzen H, de With G and Metselaar R 2001 Journal of the European Ceramic Society 21 263-268
[10] Salama M A, Rowe W M and Yasui R K 1973 NASA Technical Memorandum 33-626

[11] Endo R K, Fujihara Y and Susa M 2003 High Temperatures - High Pressures 35/36 505-511 ISSN 00181544

[12] Liu J, Yuan Y, Ren Z, Tan Q and Xiong J 2015 Sensors (Switzerland) 15 22660-22671 ISSN 14248220

[13] Kano K, Arakawa K, Takeuchi Y, Akiyama M, Ueno N and Kawahara N 2006 Sensors and Actuators, A: Physical 130-131 397-402 ISSN 09244247

[14] Zhao H and Aluru N R 2009 JOURNAL OF APPLIED PHYSICS 105 104309

[15] Jia Y and Seshia A A 2016 Journal of Microelectromechanical Systems 25 108-117 ISSN 10577157

[16] Blom F R, Bouwstra S, Elwenspoek M and Fluitman J H J 1992 Journal of Vacuum Science \& Technology B $\mathbf{1 0}$

[17] Brotz J 2004 Damping in CMOS-MEMS Resonators Tech. rep. Carnegie Mellon University

[18] Hao Z, Erbil A and Ayazi F 2003 Sensors and Actuators, A: Physical 109 156-164 ISSN 09244247

[19] Sharma A, Olszewski O Z, Torres J, Mathewson A and Houlihan R 2015 Procedia Engineering ISSN 18777058

[20] Schneider M, Bittner A and Schmid U 2015 Sensors \& Actuators: A. Physical 224 177-184 ISSN 0924-4247 URL http://dx.doi.org/10.1016/j.sna.2015.01.032 